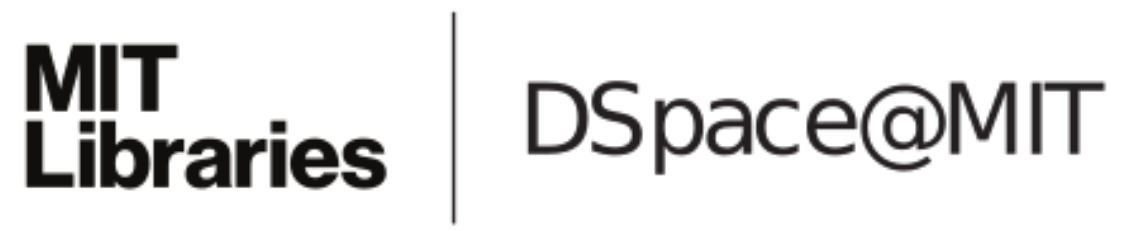

\author{
MIT Open Access Articles
}

Self-thermophoresis and thermal self-diffusion in liquids and gases

The MIT Faculty has made this article openly available. Please share how this access benefits you. Your story matters.

Citation: Brenner, Howard. "Self-thermophoresis and thermal self-diffusion in liquids and gases." Physical Review E 82.3 (2010): 036325. (c 2010 The American Physical Society

As Published: http://dx.doi.org/10.1103/PhysRevE.82.036325

Publisher: American Physical Society

Persistent URL: http://hdl.handle.net/1721.1/60399

Version: Final published version: final published article, as it appeared in a journal, conference proceedings, or other formally published context

Terms of Use: Article is made available in accordance with the publisher's policy and may be subject to US copyright law. Please refer to the publisher's site for terms of use. 


\title{
Self-thermophoresis and thermal self-diffusion in liquids and gases
}

\author{
Howard Brenner* \\ Department of Chemical Engineering, Massachusetts Institute of Technology, Cambridge, Massachusetts 02139-4307, USA
}

(Received 7 May 2010; published 30 September 2010)

\begin{abstract}
This paper demonstrates the existence of self-thermophoresis, a phenomenon whereby a virtual thermophoretic force arising from a temperature gradient in a quiescent single-component liquid or gas acts upon an individual molecule of that fluid in much the same manner as a "real" thermophoretic force acts upon a macroscopic, non-Brownian body immersed in that same fluid. In turn, self-thermophoresis acting in concert with Brownian self-diffusion gives rise to the phenomenon of thermal self-diffusion in single-component fluids. The latter furnishes quantitative explanations of both thermophoresis in pure fluids and thermal diffusion in binary mixtures (the latter composed of a dilute solution of a physicochemically inert solute whose molecules are large compared with those of the solvent continuum). Explicitly, the self-thermophoretic theory furnishes a simple expression for both the thermophoretic velocity $\mathbf{U}$ of a macroscopic body in a singlecomponent fluid subjected to a temperature gradient $\nabla T$, and the intimately related binary thermal diffusion coefficient $D_{T}$ for a two-component colloidal or macromolecular mixture. The predicted expressions $\mathbf{U}=$ $-D_{T} \boldsymbol{\nabla} T \equiv-\beta D_{S} \boldsymbol{\nabla} T$ and $D_{T}=\beta D_{S}$ (with $\beta$ and $D_{S}$ the pure solvent's respective thermal expansion and isothermal self-diffusion coefficients) are each noted to accord reasonably well with experimental data for both liquids and gases. The likely source of systematic deviations of the predicted values of $D_{T}$ from these data is discussed. This appears to be the first successful thermodiffusion theory applicable to both liquids and gases, a not insignificant achievement considering that the respective thermal diffusivities and thermophoretic velocities of these two classes of fluids differ by as much as six orders of magnitude.
\end{abstract}

DOI: 10.1103/PhysRevE.82.036325 PACS number(s): 51.10. +y, 51.20. +d, 05.20.Jj, 05.40.Jc

\section{INTRODUCTION}

\section{A. Preview}

By way of introduction to this paper, consider a nonionic binary fluid mixture (either gaseous or liquid) wherein the solute, present in only infinitesimally small concentrations, is composed of large, physicochemically inert, identical, colloidal solute molecules ("particles") or macromolecules, which together with the solvent form thermodynamically ideal solutions. When subjected to a steady temperature gradient while confined quiescently in the space between parallel walls (gravity being absent or irrelevant), solute molecules are observed to congregate near the cold wall. This behavior corresponds to one associated with the name of Soret [1,2], with the resulting steady-state inhomogeneous distribution of solute and solvent molecules regarded as reflecting a balance between the opposing forces of thermal diffusion and molecular (Fickian) diffusion. These diffusive fluxes (in generally nondilute mixtures) are, respectively, characterized by a concentration-dependent thermal diffusion coefficient $D_{T}(w)$ (which is algebraically signed) and a molecular diffusion coefficient $D(w)>0$, with the argument $w$ denoting the weight fraction of solute [2-4]. These two phenomenological coefficients constitute joint attributes of both the solute and solvent, with each coefficient possessing the same value for both species.

Consider the infinitely dilute case where $w \rightarrow 0$. The quantity $\mathbf{U}^{T}(0)$ defined by the relation

\footnotetext{
*FAX: +1 617258 8224; hbrenner@mit.edu
}

$$
\mathbf{U}^{T}(0)=-D_{T}(0) \boldsymbol{\nabla} T
$$

has the units of velocity. In the circumstances described above, where each solute molecule is so large that its Brownian motion is effectively nil (at least on a time scale sufficient for it to have migrated thermophoretically from the hot to the cold wall of the Soret apparatus), the velocity $\mathbf{U}^{T}(0)$ is generally believed in the thermodiffusion literature to be the "thermophoretic velocity" of an isolated macroscopic (i.e., non-Brownian) solute particle as it moves through the otherwise pure, solute-free solvent.

Indeed, most researchers concerned with thermal diffusion phenomena refer, indiscriminately, to thermophoresis and thermal diffusion as if the two phenomena were physically synonymous [3,5-14]. However, thermophoresis refers strictly to the motion of an isolated macroscopic (i.e., nonBrownian) particle through a single-component fluid under the influence of a temperature gradient, consequently constituting a hydrodynamic phenomenon $[15,16]$. In contrast, thermal diffusion is a nonhydrodynamic, strictly molecular phenomenon entailing (in, say, a binary mixture) the relative motion of distinct solute and solvent chemical species with respect to one another under the influence of a temperature gradient. Despite widespread belief, the velocity $\mathbf{U}^{T}(0)$ defined by Eq. (1) is not known with certainty to be the thermophoretic velocity of an isolated particle as it is tracked, experimentally, through the pure quiescent solvent, since that attribute has never before been demonstrated experimentally to hold for either for gases or liquids. This terminological schism is evident, for example, from the thermodiffusion literature on gases, where thermal diffusion [17] and thermophoresis $[15,16]$ are regarded as separate and distinct phenomena. Accordingly, for clarity, we will refer in what 
follows to $\mathbf{U}^{T}(0)$ as the mixture's "thermodiffusion" velocity (rather than its "thermophoretic" velocity), regarding it for a specified temperature gradient as a joint solute-solvent molecular property.

Inasmuch as the coefficient $D_{T}(0)$ has the same value for both solute and solvent, it follows that the thermodiffusion velocity $\mathbf{U}^{T}(0)$ defined by Eq. (1) must also be the same for both, despite the exclusive focus in the literature on this attribute as constituting a property of the solute alone. As explicitly noted above, most of the thermal diffusion literature concerned with modeling dilute solute solutions focuses on thinking of $\mathbf{U}^{T}(0)$ as being the thermophoretic velocity $[15,16]$ of a single large colloidal or macromolecular solute molecule ("particle") as it moves through the solvent. At the same time the solvent, whose molecules are assumed to be much smaller than those of the solute, is viewed as being a hydrodynamic continuum. As such, much of the current literature concerned with modeling thermal diffusion phenomena, especially in liquids [7,9-11,18-20], follows the same general approach as that used so successfully by Einstein [21] when modeling molecular (Fickian) diffusion phenomena in isothermal systems.

In marked contrast, the ansatz characterizing our model of thermal diffusion will focus on thinking of $\mathbf{U}^{T}(0)$ as the mean velocity of a single solvent molecule in the mixture, with its velocity being understood in some statisticalmolecular sense. Moreover, because we deal only with binary mixtures that are infinitely dilute in solute, and since such miniscule amounts of solute cannot, rationally, have an appreciable effect upon the behavior of the overwhelming numbers of solvent molecules present in the mixture, it is reasonable to suppose, for the same temperature gradient, that the mean velocity $\mathbf{U}^{T}(0)$ of an average solvent molecule in the mixture will not differ appreciably from its value in the absence of the solute, i.e., in the pure solvent itself.

As such, $\mathbf{U}^{T}(0)$ comes to be identified as some thermally relevant velocity of the pure solvent. However, that being the case, and using Eq. (1) in a converse, but equally "democratic" sense from that adopted originally, one is led inevitably to the view that in infinitely-dilute solutions of disparately sized molecules the thermal diffusivity $D_{T}(0)$ of the mixture must, in some approximate sense, depend only on the properties of the pure solvent [22]. This view, namely that the mixture's thermal diffusivity is largely independent of the properties of the solute, would appear to be oxymoronic relative to current modeling views [7,9-11,18-20], since the very notion of thermal diffusion seems meaningless except in connection with mixtures. After all, thermal diffusion arises solely in the context of separating the mixture's components by imposition of a temperature gradient. Yet, despite this belief, our solute-independent model of thermal diffusion will prove to be meritorious, as objectively judged by the accord of its predictions with experiment. This paper is dedicated to showing the essential truth of this counterintuitive proposition for both liquids and gases. (N.B. By common usage the term "thermal diffusion" refers only to the diffusion of species in the context of mixtures. While the diffusion of heat in single-component fluids, as given constitutively by, say, Fourier's law, also involves a diffusional process arising from a temperature gradient, that phenom- enon, termed heat conduction, does not constitute an example of "thermal diffusion.")

Explicitly, what follows is devoted to showing that the dependence of the mixture's thermal diffusivity on the physical properties of the solvent is, at least approximately, given by the expression

$$
D_{T}(0)=\beta D_{S}
$$

in which

$$
\beta=-(\partial \ln \rho / \partial T)_{p}
$$

is the solvent's coefficient of thermal expansion, wherein $\rho$ is the density and $p$ the pressure. Moreover, the solvent's selfdiffusion coefficient $D_{S}$ [23] is defined through the constitutive expression [24]

$$
\mathbf{j}^{D}=-D_{S} \boldsymbol{\nabla} \rho
$$

governing the solvent's self-diffusion flux $\mathbf{j}^{D}$ (mass per unit time per unit area) arising from local gradients in solvent density $D_{S}$ (or, equivalently, gradients in molecular number density $n=\rho / m$, with $m$ the molecular mass) due to fluctuations in an otherwise homogeneous, isothermal fluid.

To the extent that Eq. (2) can be shown to be correct, Eq. (1) then furnishes the mixture's thermodiffusion velocity $\mathbf{U}^{T}(0)$ (and conversely).

\section{B. Mass velocity vs volume velocity}

Before proceeding, it remains to resolve the philosophical conundrum whereby the mixture in a Soret experiment is at rest, and yet both solute and solvent move with the same nonzero velocity, $\mathbf{U}^{T}(0)$. Resolution of this dilemma resides in the existence of two independent experimentally based definitions of what is meant, physically, by the "velocity" of a fluid. One of these two continuum fluid velocities, designated by the symbol $\mathbf{v}_{m}$, pertains to the movement of mass. The other, designated by the symbol $\mathbf{v}_{v}$, pertains to the movement of volume [25]. In the past, these two velocities have always been implicitly regarded as synonymous, at least for single-component fluids [26-29] under all circumstances, but without formal proof. These two velocities will, however, be seen in what follows as unequal in the present nonisothermal fluid case [24,28,29] (though equal in the commonly encountered isothermal case).

The fundamental physical notion of velocity entering into the basic equations of fluid mechanics involves the movement of mass. Specifically, the definition of the fluid's conventional (kinematical) mass velocity $\mathbf{v}_{m}$ at a point of the fluid is such that it appears in the continuity equation $\partial \rho / \partial t$ $+\boldsymbol{\nabla} \cdot\left(\rho \mathbf{v}_{m}\right)=0$ expressing the law of conservation of mass. However, the more fundamental notion of velocity is geometric rather than kinematic, being obtained experimentally by monitoring the temporal movement of a discrete pointsize object as it moves through space. In that case the object's mass is irrelevant to the definition of velocity.

Experimentally, there are two independent ways of measuring a single-component fluid's continuum "velocity" at a specified fluid point, say $P$ : (i) When the fluid is photochromic, one focuses a laser beam for an instant at a point 
slightly upstream of $P$, and subsequently tracks the movement of the resulting blob of color as it moves downstream, passing through $P$. Since this color is carried by the molecules of the fluid, and since these same molecules also serve as the carriers of the fluid's mass, it is obvious that this type of velocity measurement furnishes $\mathbf{v}_{m}$. (Modulo some uncertainty, use of a dye for the case where the fluid is not photochromic constitutes an equivalent scheme); (ii) the second scheme involves introducing a small, albeit nonBrownian, physicochemically passive particle into the fluid upstream of $P$, and subsequently monitoring the spatiotemporal movement of this particulate "tracer" as it passes through $P$. This protocol [30] constitutes a purely geometric measurement of the fluid's velocity, since it is unrelated to the concept of mass.

It appears to be universally accepted in books on hydrodynamics [31-33] that these two velocity scenarios, which differ only in the types of "tracer" used, molecular or material, furnish identical values of the fluid's velocity. While this appears to be true for isothermal fluids, velocity measurements in nonisothermal fluids point to a difference arising as a consequence of the nonBrownian tracer's thermophoretic motion $[15,16]$. The most dramatic illustration thereof arises in the case of a quiescent fluid subjected to a steady temperature gradient, where a photochromic tracer is observed to remain in place, while a particulate tracer is observed to move from hot to cold as a result of the phenomenon of thermophoresis.

Thermophoresis is a well documented phenomenon in the case of gases $[15,16]$, where thermophoretic velocities are vastly larger than for liquids. The main finding of such measurements is that, for a given gas (i.e., "solvent") the particle's thermophoretic velocity is roughly the same for all particles, independent of their size, shape, and physicochemical properties relative to those of the gas, at least for the case of thermally inert (i.e., nonheat-conducting) particles [34]. This relative lack of dependence of the particle's thermophoretic velocity $\mathbf{U}$ upon all of the particle's physical and chemical properties supports our claim that the solute's thermodiffusion velocity $\mathbf{U}^{T}(0)$, defined by Eq. (1), is closely related to the solute's thermophoretic velocity $\mathbf{U}$, and hence to the pure solvent "s "self-thermophoretic" velocity, at least in the case of gases. The case for liquids is more tenuous (see Sec. VII C) because of the profound difficulties that arise when attempting to directly measure the miniscule thermophoretic velocities encountered.

More quantitatively, Eqs. (1) and (2) in combination show that the mixture's thermodiffusion velocity, when expressed in terms of the pure solvent's properties, is given by the expression

$$
\mathbf{U}^{T}(0)=-\beta D_{S} \nabla T
$$

a result that will be noted in Secs. II and III to accord well with thermophoretic particle velocity experiments performed with gases. That is, with $\mathbf{U}^{T}(0)$ defined by Eq. (5) rather than Eq. (1) the relation $\mathbf{U} \approx \mathbf{U}^{T}(0)$ is found to be reasonably well confirmed for gases.

However, apart from any and all considerations of velocity, whether thermodiffusive or thermophoretic, Eq. (2) for the thermal diffusivity will be seen in what follows to be reasonably well supported for many different liquid mixtures (as well as for the smaller number of gaseous mixtures thus far investigated) [22].

Independently of quantitative issues and even of specific interest in thermodiffusion phenomena, the present work confirms on purely phenomenological grounds that the molecules of a quiescent single-component fluid when subjected to a temperature gradient possess a volumetric velocity $\left(\mathbf{v}_{v}\right.$ $\neq \mathbf{0})$ despite lacking a mass velocity $\left(\mathbf{v}_{m}=\mathbf{0}\right)$. (The appendix to this paper elaborates, briefly, on the physical meaning of the volume velocity in relation to the fluid's mass velocity.) Objective evidence of the existence of these two independent quantities, both constituting equally rational candidates for designation as the fluid's "velocity," is seen to derive from the outcomes of different types of tracer experiments. Among other things this bivelocity phenomenon [24] appears to provide clear and unequivocal evidence of the ability of single-component liquids and gases to transport macroscopic fluid volume by molecular motion (Brownian motion) alone, without concomitant macroscopic mass motion. If indeed true, this resolves the presently contentious issue surrounding the existence of diffuse volume motion in singlecomponent fluids [24] (there being no doubt of its existence in the case of mixtures [24,28,29]).

\section{Single-component fluids}

Self-diffusion $[35,36]$ is a Brownian motion-animated phenomenon occurring in a quiescent, single-component, isothermal, external force-free fluid of uniform density wherein, macroscopically speaking, nothing overtly happens to the fluid at a hydrodynamic level of description as a result of the self-diffusion process. Yet, as is well known, selfdiffusion constitutes a phenomenon that is macroscopically observable by tracking the spatiotemporal movement of a collection of marked molecules of the fluid [35].

"Self-thermophoresis" [37] is, similarly, a phenomenon occurring in a quiescent, single-component fluid, albeit one now subjected to a steady temperature gradient [rendering the fluid's density nonuniform owing to the uniformity of the pressure considered in conjunction with the fluid's equation of state, $\rho=\rho(T, p)]$. Again, macroscopically speaking, nothing overtly happens to the fluid as a whole as a result of this phenomenon. Yet, it too constitutes a phenomenon whose existence can, in principle, be rendered observable by tracking the spatiotemporal movement of a marked molecule of the fluid.

Explicitly, such a molecule will-owing to its Brownian motion acting in concert with the self-thermophoretic (or "thermoösmotic") force exerted upon it as a consequence of the steady temperature gradient [37]—-move, on average, toward the region of increasing density (and concomitantly decreasing temperature when $\beta>0$ ) rather than remaining in place. This biased Brownian motion contrasts with the preceding case of self-diffusion occurring in isothermal fluids, whose uniform density renders all positions in the fluid equally probable, and thus bias-free, such that, on average, a tracer molecule remains permanently in place. This direc- 
tionally biased movement of a molecule in a nonisothermal fluid constitutes the microscopic counterpart of the thermophoretic movement of a macroscopic body in such a fluid $[15,16]$. It is this analogy that renders the appellation "selfthermophoresis" appropriate.

Intimately related thereto is the closely related phenomenon of "thermal self-diffusion" in single-component fluids, referring to a flux of molecules arising as a result of a temperature gradient, and representing the collective selfthermophoretic transport of individual molecules. This flux constitutes for the single-component solvent the analog of the thermal diffusion flux of a chemical species occurring in a mixture as a consequence of a temperature gradient. Pursuing an analogy with the definition of the thermal diffusion coefficient $D_{T}$ in a binary mixture [2-4], the constitutive equation for the pure fluid's thermal self-diffusion mass flux $\mathbf{j}^{T}$ of molecules in isobaric circumstances is taken to be

$$
\mathbf{j}^{T}=-\rho D_{T}^{S} \boldsymbol{\nabla} T .
$$

By definition, $D_{T}^{S}$ is termed the single-component fluid's thermal self-diffusion coefficient (or, less elegantly, its selfthermal diffusion coefficient). It is an algebraically signed scalar. When the direction of the flux is from hot to cold, which is generally the case, we have that $D_{T}^{S}>0$. Conversely, in the opposite case, $D_{T}^{S}<0$.

In near-equilibrium circumstances, it is shown in what follows that this coefficient is given constitutively by the simple expression

$$
D_{T}^{S}=\beta D_{S} .
$$

In contrast with the approximate Eq. (2) for dilute mixtures this is an exact relation since it is effectively true by definition.

\section{Binary mixtures}

For the case of a thermodynamically ideal two-component mixture composed of a dilute dispersion of large, unconstrained, physicochemically inert solute molecules (colloidal or macromolecular) dispersed or dissolved in a solvent continuum it is shown in what follows that the mixture's binary thermal diffusion coefficient $D_{T}(0)$ in solutions that are infinitely-dilute in the solute is, at least approximately, identical to that of the pure solvent,

$$
D_{T}(0)=D_{T}^{S}
$$

Semenov and Schimpf [20] present a theory of thermal diffusion in binary liquid mixtures. In the course of their review of the pertinent literature they state that "... the "thermophoretic' motion of a pure liquid is impossible according to the laws of formal logic," thereby dismissing arguments embodied in an alternative thermal diffusion theory proposed by the writer [22] based on the phenomenon of selfthermophoresis. In that context those authors go on to claim, as a consequence, that only their model can rationally explain the role played by the coefficient of thermal expansion in establishing the direction of phoretic migration. It is shown here, contrary to the Semenov-Schimpf claim, that self-thermophoretic forces do indeed exist in pure fluids.
Moreover, as suggested by Eq. (2), together with the latter's applicability to binary mixtures of disparately-sized molecules, such self-thermophoretic phenomena prove fundamental to a new approach to transport processes in nonisothermal fluids involving the notion of diffuse volume transport $[24,38,39]$. In validating these claims, attention is initially confined for simplicity to the case of ideal gases, with liquids being treated later.

\section{SELF-THERMOPHORETIC MOLECULAR FORCES AND VELOCITIES IN PURE GASES}

\section{A. Experimental configuration}

Consider a quiescent single-component ideal gas subject to the action of gravity while confined within the region 0 $<x<L$ bounded between two indefinitely extended horizontal plates separated by a distance $L$. The plates, situated at $x=0$ and $x=L$, are permanently maintained at the respective hot and cold temperatures $T_{h}$ and $T_{c}\left(T_{h}>T_{c}\right)$, with the hotter plate situated below the colder one. The confined gas is assumed to undergo a steady, one-dimensional heat-conduction process where, despite the presence of gravity, the gas remains quiescent, free of natural convection. By virtue of the gas being at rest it follows that $\mathbf{v}_{m}=\mathbf{0}$.

Attention is further limited to situations for which the temperature difference between the two plates is relatively small, such that $\gamma:=\left(T_{h}-T_{c}\right) / T_{o} \ll 1$ for $T_{o}$ any temperature lying in the range $T_{c}<T_{o}<T_{h}$. In such circumstances, local variations in the gas's thermal conductivity $k$ may be neglected. Accordingly, the (algebraically signed) temperature gradient may be regarded as essentially uniform throughout the gas at the value $d T / d x=-\left(T_{h}-T_{c}\right) / L=$ const, with the temperature field satisfying the steady-state energy equation $d^{2} T / d x^{2}=0$, and thus possessing the solution $T(x)=T_{h}-\left(T_{h}\right.$ $\left.-T_{c}\right) x / L$.

In accord with the configuration described above, gravity acts in the same direction as the temperature gradient, namely, in the negative $x$ direction, so that with $\mathbf{g}$ Earth's gravity vector it follows that $\mathbf{g}=-\hat{\mathbf{x}} g$. Here, $\hat{\mathbf{x}}$ is a unit vector in the positive $x$ direction and $g>0$ is the magnitude of the gravity field. While this configuration is potentially unstable due to the colder fluid being on top, instability does not set in until the magnitude $\left(T_{h}-T_{c}\right) / L$ of the temperature gradient exceeds a certain critical value attained at a Rayleigh number of $\mathrm{Ra}=g L^{3}|\beta| \Delta / v \alpha=1708$. This dimensionless number constitutes the threshold value for the onset of convection in the classical Rayleigh-Bénard problem [40]. For ideal gases $\beta$ $=1 / T$. Additionally, $\Delta=T_{h}-T_{c}$ denotes the temperature difference, while $v=\eta / \rho$ is the kinematic viscosity (with $\eta$ the shear viscosity), and $\alpha=k / \rho \hat{c}_{p}$ is the thermometric diffusivity [33], in which $\hat{c}_{p}$ is the isobaric specific heat capacity. We suppose that $|\beta| \Delta \equiv \gamma \ll 1$, thus assuring hydrodynamic stability of the quiescent state for physically reasonable choices of system parameters - a fact that will subsequently be confirmed.

\section{B. Uniform density case, $\rho=$ const}

Were the fluid isothermal the action of gravity would be such as to cause the confined gas to be densest at the bottom 
plate $x=0$. On the other hand were the fluid nonisothermal and gravity absent, the gas would then be densest at the cold upper plate, $x=L$. As such, in the present case, where gravity and temperature gradients act concurrently, each effect tends to offset the other. As a consequence it will be seen for a specified gas and for a given plate spacing $L$ that it is possible to choose the temperature difference $\Delta$ such that the gas's density is uniform throughout the entire gap region, 0 $<x<L$. We focus in this section on that case.

For the present steady-state quiescent heat-conduction process the hydrostatic equation requires that

$$
\nabla p=\rho \mathbf{g} .
$$

Thus, when $\rho$ is independent of position (and $\mathbf{g}$ is taken to be constant, independent of vertical position $x$ ), it follows that the local pressure gradient $d p / d x$ is constant throughout the gas, possessing the value $d p / d x=-\left(p_{h}-p_{c}\right) / L=-\rho g=$ const.

With $R$ the universal gas constant and $M_{w}$ the gas's molecular weight it follows from the ideal gas law,

$$
p=\frac{R}{M_{w}} \rho T,
$$

that for the present uniform density case the required temperature gradient must be of magnitude

$$
\frac{T_{h}-T_{c}}{L}=g \frac{M_{w}}{R} .
$$

For example, in the case of hydrogen $\left(M_{w}=2.016 \mathrm{~kg} / \mathrm{kg}\right.$ - mol) with $g=9.81 \mathrm{~m} / \mathrm{s}^{2}$ for Earth's gravity field, and $R$ $=8.315 \times 10^{-3} \mathrm{~kg} \mathrm{~m}^{2} / \mathrm{s}^{2} \mathrm{~kg}-\mathrm{mol} \mathrm{K}$, this yields a temperature gradient of $2.40 \times 10^{-3} \mathrm{~K} / \mathrm{m}$. For a gap of, say, $L$ $=10 \mathrm{~cm}$, and for a mean temperature of $T_{o}=300 \mathrm{~K}$ (at which temperature $v=1.095 \times 10^{-4} \mathrm{~m}^{2} / \mathrm{s}$ and $\alpha=1.554$ $\times 10^{-4} \mathrm{~m}^{2} / \mathrm{s}$ [41]) this yields a Rayleigh number of $\mathrm{Ra}$ $=0.46$. This is well below the critical threshold at which convective instability would be initiated. As such, the proposed configuration of "cold above hot" is seen to be stable in gases over a wide range of operating conditions.

\section{Self-thermophoretic force on a molecule}

Upon converting from molar to mass units using $R=k_{b} N$ and $M_{w}=m N$, where $N$ is Avogadro's number, $k_{b}$ is Boltzmann's constant, and $m$ is the mass of a molecule, it follows that Eq. (11) can be rewritten locally as

$$
\boldsymbol{\nabla} T=\frac{m}{k_{b}} \mathbf{g} .
$$

As the external force acting on a single molecule of the gas is

$$
\mathbf{F}=m \mathbf{g}
$$

one finds upon eliminating $\mathbf{g}$ between the last two equations that

$$
\mathbf{F}=k_{b} \nabla T \text {. }
$$

The latter force tends to cause the molecule to move downward, in the direction of the temperature gradient.
However, statistically speaking, the molecule does not move in response to that force; rather, on average, it remains in place, corresponding to the fact that the gas is macroscopically quiescent $\left(\mathbf{v}_{m}=\mathbf{0}\right)$. As a consequence, $\mathbf{F}$ must, on average, be opposed by an equal and opposite force,

$$
\mathbf{F}^{T}=-k_{b} \nabla T \text {. }
$$

This virtual force, which tends to move the molecule from the hotter toward the colder temperatures, will henceforth be called the self-thermophoretic force. It should be regarded as "hydrodynamic" in origin since it constitutes a statistical force exerted on the molecule by virtue of the presence of all of the other confined molecules in the system. (The word "hydrodynamic" as used here refers to the fluid's nonzero volume motion $\mathbf{v}_{v} \neq \mathbf{0}$ arising as a consequence of the temperature gradient.)

\section{Virtual thermophoretic velocity}

Were the molecule not constrained, on average, to remain in place, the tendency of this self-thermophoretic force would be to cause the molecule to move upward through the quiescent fluid with a velocity

$$
\mathbf{U}^{T}=M \mathbf{F}^{T},
$$

in which $M$ is the molecule's hydrodynamic mobility. According to the Nernst-Planck-Einstein equation this mobility is related to the gas's self-diffusion coefficient $D_{S}$ by the expression [21]

$$
M=D_{S} / k_{b} T .
$$

Equations (15)-(17) combine to yield the expression

$$
\mathbf{U}^{T}=-D_{S} \nabla \ln T .
$$

Alternatively, with

$$
\mathrm{Sc}=v / D_{S}
$$

the Schmidt number [33] we have that

$$
\mathbf{U}^{T}=-\mathrm{Sc}^{-1} v \boldsymbol{\nabla} \ln T .
$$

This defines the gas's self-thermophoretic velocity, which, like $\mathbf{F}^{T}$, is virtual.

\section{E. Magnitudes of the self-thermophoretic forces and velocities}

Use of the hydrogen example cited above together with Eq. (15) for the thermophoretic force shows that even a very modest temperature gradient of $1 \mathrm{~K} / \mathrm{m}$ is equivalent to 417 gees, with 1 gee the magnitude of Earth's gravity. Obviously, even for very modest temperature differences, the use of small gap widths (such as in the case of thermal field-flow fractionation [42]) enables one to easily achieve forces millions of time stronger than that of gravity. This shows why thermal diffusion is competitive with that of ultracentrifugation as a separation process for species of different molecular weights.

Using the value $D_{S}=1.4 \times 10^{-4} \mathrm{~m}^{2} / \mathrm{s}$ for the selfdiffusivity of hydrogen at room temperature $[[17]$, p. 267] 
and a temperature gradient of $1 \mathrm{~K} / \mathrm{m}$, the self-thermophoretic velocity of a hydrogen molecule is, according to Eq. (18), $U^{T}=3 \times 10^{-7} \mathrm{~m} / \mathrm{s}$. While this number appears quite modest, that is due to the extremely small temperature gradient used in its calculation. At realistically achievable temperature gradients, such as in the case of thermal field-flow fractionation [42], speeds can arise that are of macroscopic interest in terms of the time scales pertinent to thermal diffusion separation phenomena.

\section{COMPARISON WITH EXPERIMENTALLY MEASURED THERMOPHORETIC VELOCITIES IN PURE GASES}

Experimentally, when a macroscopic, non-heatconducting (or insulated) rigid non-Brownian particle is inserted into an otherwise quiescent, single-component ideal gas continuum undergoing steady-state heat conduction in the absence of gravity, the particle is observed to move through the gas with a velocity given by the expression $[34,43]$

$$
\mathbf{U}=-C_{S} v \nabla \ln T .
$$

$C_{S}$ is a dimensionless parameter of $O(1)$ termed Maxwell's thermal creep coefficient [44], whose value can only be established experimentally. This phenomenon, whereby a solid body of macroscopic dimensions moves though a fluid solely as a result of a temperature gradient, is termed thermophoresis $[15,16]$, and its velocity $\mathbf{U}$ is said to be the particle's thermophoretic velocity. In addition to its experimental basis, Eq. (21) also has a theoretical basis [34,43].

The constitutive formula (21) holds independently of the particle's size and shape, as well as of the particle's orientation relative to the temperature gradient, these being theoretical facts [34] confirmed by experiment. Experiments further reveal that $C_{S}$ is insensitive to the physiochemical constitution of either the gas or particle, again for the case of nonheat-conducting particles.

Though Maxwell [44] originally estimated his thermal creep coefficient to have the value $3 / 4$, gaseous thermophoretic experiments by Talbot, et al. [45] cite the "best-fit" value to be, approximately,

$$
C_{S} \approx 1.17 \text {. }
$$

On the other hand, for the case of a dilute monatomic gas whose molecules are assumed to interact via elastic, hardsphere collisions-corresponding to an infinitely-repulsive inverse power intermolecular potential-one has that $[[17(\mathrm{a})]$, p. $275 ;[46]]$

$$
\mathrm{Sc}^{-1} \approx 1.20 \text {. }
$$

That the last numbers are close to another is of theoretical import.

\section{A. Equality of solute- and solvent-molecule thermophoretic velocities}

Comparison of Eq. (20) with Eq. (21) in light of the values of the respective $C_{S}$ and $\mathrm{Sc}^{-1}$ coefficients noted above leads to the seemingly inescapable conclusion that

$$
\mathbf{U}^{T}=\mathbf{U},
$$

at least to a high degree of approximation. The equality (24) shows the thermophoretic velocity $\mathbf{U}$ of a non-heatconducting macroscopic body to be identical to the selfthermophoretic velocity $\mathbf{U}^{T}$ of a molecule of the pure gas. It is this equality that leads to adoption of the appellation "selfthermophoresis" [47] when applied to the fluid.

\section{B. Volume velocity}

The credibility of the empirical conclusion embodied in Eq. (24) is further enhanced by the independent fact that the hydrodynamic volume velocity $\mathbf{v}_{v}$ [25] of a quiescent singlecomponent fluid, whether liquid or gas, is the same as its diffuse volume velocity $\mathbf{v}_{v}=\mathbf{j}_{v}$, with the latter given by the general expression [24]

$$
\mathbf{j}_{v}=-D_{S} \beta \boldsymbol{\nabla} T+D_{S} \kappa(\nabla p-\rho \mathbf{g}),
$$

where $\kappa=(\partial \ln \rho / \partial p)_{T}$ is the fluid's isothermal compressibility coefficient (which for a dilute gas is $\kappa=1 / p$ ). Thus, for the present hydrostatic case, where Eq. (9) applies, and for the case of ideal gases, where $\beta=1 / T$, one obtains $\mathbf{j}_{v}^{T}=$ $-D_{S} \boldsymbol{\nabla} \ln T$, wherein the superscript $T$ refers to that portion of the diffuse volume flux $\mathbf{j}_{v}$ arising solely as a consequence of the temperature gradient. Equivalently,

$$
\mathbf{j}_{v}^{T}=-\mathrm{Sc}^{-1} v \boldsymbol{\nabla} \ln T,
$$

a result identical to that for the gas's thermophoretic velocity, Eq. (20). Consequently, we conclude that

$$
\mathbf{U}^{T}=\mathbf{j}_{v}^{T} .
$$

The interpretation of the preceding identity is that, despite the gas being macroscopically at rest, there is, nevertheless, a diffuse upward "flow" of gas volume, from hot to cold, tending to entrain within it the molecules of the gas (which, of course, remain stationary on average owing to the opposing action of gravity). This balancing of drag and gravity forces acting on a body (a molecule in this case) defines a condition that in the terminology of suspension hydraulics for macroscopic particles is called the "teeter" condition [48].

\section{MOLECULAR SELF-THERMOPHORESIS AND THERMAL SELF-DIFFUSION IN PURE LIQUIDS}

This section extends the arguments of the preceding section to liquids. However, a different approach is used here, wherein we no longer begin by addressing the case where the density of the fluid is uniform throughout. Nor do we assign any role in the derivation to gravity. Whereas the analysis that follows in this section focuses on liquids, it will, owing to its generic nature, be seen as applicable to gases as well.

Consider again the case of steady-state heat conduction in a quiescent fluid, which now may be either liquid or gas. As before, the fluid is confined between two plates maintained at different temperatures. In the present case, owing to the absence of gravity, and the consequent uniformity of the pressure throughout, the density at a point of the fluid will now 
vary with the local temperature at that point as given by the fluid's equation of state,

$$
\rho=\rho(T), \quad(p=\text { const }) .
$$

At least for the case where $\beta>0$ the density of the fluid will be greatest in the region near the cold wall. The molecular interpretation of this fact is that a self-thermophoretic force acts to drive a molecule toward that wall, thus increasing the probability of its being present in that region, and hence increasing the density in that region. On the other hand the fluid's ever-present Brownian motion acts in such a direction as to tend to create a state of uniform density, where the molecules are distributed homogeneously throughout the fluid. Thus (again for case where $\beta>0$ ), Brownian motion acts to drive molecules away from the colder wall. The steady-state distribution of molecules existing between the plates can thus be viewed as a dynamic steady state, one in which the self-thermophoretic flux $\mathbf{j}^{T}$ of molecules toward the cold wall is balanced by a Brownian diffusion flux $\mathbf{j}^{D}$ of molecules away from that wall.

Explicitly, for the present steady-state heat-conduction case, and with $\mathbf{j}^{T}$ and $\mathbf{j}^{D}$ defined as in Eqs. (6) and (4), respectively, the net mass flux,

$$
\mathbf{j}=\mathbf{j}^{T}+\mathbf{j}^{D}
$$

is necessarily identically zero. Consequently, we obtain $D_{T}^{S} \boldsymbol{\nabla} T=-D_{S} \boldsymbol{\nabla} \ln \rho$. With use of Eqs. (6) and (28) it follows in the present constant pressure case that $\nabla \ln \rho=-\beta \nabla T$. The latter pair of equations combine so as to confirm Eq. (7).

Instead of regarding the kinematical flux equation as the source of Eq. (7), one could, alternatively, also regard this expression as resulting from a force balance between a virtual self-phoretic thermal force $\mathbf{F}^{T}$ and a virtual Brownian diffusion force $\mathbf{F}^{D}$, such that the net force is identically zero: $\mathbf{F}=\mathbf{F}^{T}+\mathbf{F}^{D}=\mathbf{0}$. Upon defining the virtual velocities $\mathbf{U}^{T}$ and $\mathbf{U}^{D}$ in relation to the preceding fluxes as $\mathbf{j}^{i}=\mathbf{U}^{i} \rho(i=T, D)$, together with use of Eqs. (6) and (4), this yields

$$
\mathbf{U}^{T}=-D_{T}^{S} \boldsymbol{\nabla} T
$$

and

$$
\mathbf{U}^{D}=-D_{S} \boldsymbol{\nabla} \ln \rho .
$$

In conjunction with the definitions $\mathbf{U}^{i}=M \mathbf{F}^{i}(i=T, D)$ in which $M$ is given by Eq. (17), these make

$$
\mathbf{F}^{T}=-k_{b} T\left(D_{T}^{S} / D_{S}\right) \nabla T
$$

and

$$
\mathbf{F}^{D}=-k_{b} T \nabla \ln \rho .
$$

As the sum of these two forces is zero, use of Eq. (6) in this sum confirms Eq. (7).

For later reference we note that the introduction of Eq. (7) into Eq. (30a) gives

$$
\mathbf{U}^{T}=-D_{S} \beta \boldsymbol{\nabla} T=\mathbf{U}^{D} .
$$

In the case of gases this agrees with Eq. (18). Comparison with Eq. (25) for the present hydrostatic equilibrium case shows that

$$
\mathbf{U}^{T}=\mathbf{j}_{v}^{T}=\mathbf{U}^{D},
$$

the first member of these two equalities having already been given in Eq. (27), albeit only for the case of gases.

\section{V. (MACROSCOPIC) THERMOPHORESIS IN PURE LIQUIDS}

In seeking to confirm Eq. (32) by comparing its predictions against experimental data it needs to be borne in mind that this formula constitutes a purely self-thermophoretic result. That is, there remains the open question as to whether Eq. (32) for $\mathbf{U}^{T}$ would correctly describe the experimentally observed thermophoretic velocity $\mathbf{U}$ of a macroscopic (nonheat-conducting) body moving quasistically through a liquid under the influence of a temperature gradient. While this question was positively resolved in the case of gases by demonstrating accord between Eqs. (20) and (21) we are unaware that a noncontroversial counterpart of the experimental correlation [Eq. (21)] exists for liquids.

Indeed, there exists a dearth of data for the thermophoretic velocities of macroscopic particles moving through liquids, certainly compared with the relative abundance of such data for gases. This difference can be traced to the enormous difference in the values of the self-diffusion coefficients for liquids [35] vs those for gases [[17(a)], p. 267]. For example, the self-diffusivity of liquid water at $25^{\circ} \mathrm{C}$ is $D_{S}=2.30 \times 10^{-9} \mathrm{~m}^{2} \mathrm{~s}^{-1}$, whereas that for gaseous hydrogen at roughly the same temperature $\left(23^{\circ} \mathrm{C}\right)$ is $D_{S}=1.46$ $\times 10^{-4} \mathrm{~m}^{2} \mathrm{~s}^{-1}$. The respective coefficients of thermal expansion at these same temperatures are [22] $\beta=0.26$ $\times 10^{-3} \mathrm{~K}^{-1}$ for water and $\beta=3.4 \times 10^{-3} \mathrm{~K}^{-1}$ for hydrogen. Consequently, according to Eq. (33), for the same temperature gradient the self-thermophoretic velocity $\mathbf{U}^{T}$ of a water molecule would be roughly six orders-of-magnitude less than that of a hydrogen molecule. To the extent that the experimental thermophoretic velocity $\mathbf{U}$ of a macroscopic body through a liquid was roughly the same as the liquid's selfthermophoretic velocity $\mathbf{U}^{T}$, as we have shown to be true in the case of gases [cf. Eq. (24)] [and whose validity is implied in Sec. VII C addressed to liquids], a particle moving through water would move a million times more slowly than it would through hydrogen for the same temperature gradient.

Given the known experimental difficulties encountered when measuring the thermophoretic velocity $\mathbf{U}$ of a particle in a gas [49], especially as a consequence of gravity and natural convection, it would appear extremely difficult to directly measure the thermophoretic velocity of a macroscopic particle in a liquid by single-particle tracking, although claims to that effect have been made [as discussed in Sec. VII C (subsection thermophoretic velocities in liquids)]. On the other hand, when the particles are of colloidal or macromolecular dimensions, indirect measurements are possible, based either on the arguments of the next paragraph, or on Eq. (37). This experimental issue is subsequently addressed.

While direct measurement of a macroscopic particle's thermophoretic velocity $\mathbf{U}$ in a liquid appears fraught with problems, an indirect experimental measurement (more ac- 
curately, an estimate) of the particle-free liquid's selfthermophoretic velocity $\mathbf{U}^{T}$ is surprisingly easy, despite being virtually infinitesimally small. The ability to measure that velocity is linked to the fundamental role it plays in an elementary model of the Soret effect (see Sec. VI) pertaining to thermal diffusion in binary liquid mixtures [1-4], when this role is considered in conjunction with the fact that $\mathbf{j}_{v}^{T}$ $=\mathbf{U}^{T}$, as in Eq. (27). Use of that Soret model involves indirectly measuring the solvent's volume velocity $\mathbf{v}_{v}$, viewed as a flux of volume, rather than as the (mean) thermophoretic velocity $\mathbf{U}^{T}$ of a single molecule of the solvent. A review of experimental data confirming that this Soret-based model does indeed furnish a reliable estimate of the solvent's volume velocity $\mathbf{v}_{v} \equiv \mathbf{j}_{v}^{T}$, and hence of $\mathbf{U}^{T}$, is discussed in a recent paper [24]. What follows below independently confirms these statements.

\section{THERMAL DIFFUSION IN BINARY MIXTURES}

\section{A. Physical basis of the model}

This section provides a derivation of the constitutive formula (2) for the binary thermal diffusion coefficient $D_{T}(0)$ in a two-component liquid or gaseous mixture composed of large, physicochemically inert, external force-free solute molecules dissolved or dispersed in a solvent continuum, in the limit where the mixture is infinitely dilute in solute. The resultant finding reproduces an expression given in an earlier paper [22] addressed to this same idealized set of solutesolvent circumstances. However, that derivation was based on the ad hoc assumption that the mixture's volume diffusivity $D_{v}$ appearing in the constitutive equation [22]. $\mathbf{j}_{v}$ $=D_{v} \boldsymbol{\nabla} \ln \rho$ for the mixture's diffuse volume flux $\mathbf{j}_{v}$ [28] was equal to the pure solvent's self-diffusion coefficient $D_{S}$. Here, the assumption that $D_{v}=D_{S}$ is proved. At the same time, in Sec. VII we review the experimental data in support of our constitutive Eq. (2) for $D_{T}(0)$, including limitations imposed by the assumption of physicochemical inertness when interpreting these data.

There are several different approaches to the derivation, each of which entails use of a different physical model, but all of which lead to the same end result, namely, Eq. (2). Each model hinges upon the dominant fact that for a given temperature gradient, and in the infinitely dilute solute limit where the fluid in the binary mixture consists of virtually pure solvent, the flux of solvent molecules in the mixture is the same as that in the pure solvent. The validity of this argument can be seen by considering identical Soret-type steady-state experiments performed on quiescent fluids in confined spaces, one experiment performed with the infinitely dilute mixture present and the other performed with the pure solvent appearing in its place. Because of the solute's diluteness it is obvious that in the limit the spatial distribution of solvent molecules, as well as their other attributes, such as their thermal agitation velocities, will be virtually identical in the two experiments. As such, the known properties of the pure solvent molecules attributable to the temperature gradient, which is the driving force common to both experiments, can also be assigned to the solvent molecules present in the mixture.

\section{B. Two different, but equivalent, models}

In the first instance the argument eventually leading to Eq. (2) is simply that (for the same applied temperature gradient) the thermophoretic velocity $\mathbf{U}^{T}(0)$ of the solvent molecules in the mixture [50] will be equal to the self-thermophoretic velocity $\mathbf{U}^{T}$ of the solvent molecules in the pure fluid, so that

$$
\mathbf{U}^{T}(0)=\mathbf{U}^{T} \text {. }
$$

This equality reflects "democratic" reasoning of the type cited in the Preview whereby the velocity of the solvent is necessarily the same as that of the solute molecules in the mixture, as given by Eq. (1)

Use in the above of Eq. (1) for $\mathbf{U}^{T}(0)$ and Eq. (32) for $\mathbf{U}^{T}$ leads immediately to the relation

$$
D_{T}(0)=\beta D_{S},
$$

in accord with Eq. (2).

An alternative approach to proof of Eq. (35) based upon volume transport rather than thermophoresis necessitates that we first review the general constitutive equations governing the diffuse fluxes of the solute (species 1) and solvent (species 2) in the mixture, these being (in the absence of both pressure gradients and external forces) [1-4]

$$
\mathbf{j}_{\alpha}=\mathbf{j}_{\alpha}^{T}+\mathbf{j}_{\alpha}^{D}, \quad(\alpha=1,2) .
$$

Here, $\mathbf{j}_{\alpha}$ is the diffuse mass flux of species $\alpha$. In the above $[1-4,27]$,

$$
\begin{gathered}
\mathbf{j}_{1}^{T}=-\rho w(1-w) D_{T} \boldsymbol{\nabla} T \\
\mathbf{j}_{2}^{T}=\rho w(1-w) D_{T} \boldsymbol{\nabla} T
\end{gathered}
$$

and

$$
\begin{gathered}
\mathbf{j}_{1}^{D}=-\rho D \nabla w, \\
\mathbf{j}_{2}^{D}=\rho D \nabla w .
\end{gathered}
$$

As before, $D_{T}(w)$ is the binary thermal diffusivity, $w$ is the weight fraction of solute, and $D(w)$ is the mutual diffusion coefficient [51]. In the infinitely dilute solute limit $w \ll 1$ we note for later reference that Eq. (37a) becomes

$$
\mathbf{j}_{1}^{T}(0)=-\rho w D_{T}(0) \nabla T \text {. }
$$

For the same temperature gradient, and as a consequence of the solute's diluteness together with the absence of mass motion in the quiescent Soret experiment, it follows that the diffuse flux of volume (volume per unit area per unit time) through the Soret apparatus attributable to the temperate gradient alone, namely $\mathbf{j}_{v}^{T}(0)$, must be the same as the diffuse flux of volume through the apparatus when only the pure, solute-free solvent is present, namely, $\mathbf{j}_{v}$. Consequently,

$$
\mathbf{j}_{v}^{T}(0)=\mathbf{j}_{v}^{T} .
$$

However, according to Eq. (25), $\mathbf{j}_{v}^{T}=-\beta D_{S} \boldsymbol{\nabla} T$ for the hydrostatic case, whence

$$
\mathbf{j}_{v}^{T}(0)=-\beta D_{S} \nabla T
$$

Since $\rho w$ is the mass of solute per unit volume in the mixture, it follows that the hypothetical mass flux of solute 
through the Soret apparatus (in the absence of molecular diffusion) arising from the temperature gradient is

$$
\mathbf{j}_{1}^{T}(0)=\rho w \mathbf{j}_{v}^{T}(0) \text {. }
$$

Introduction of Eq. (41) into the latter gives

$$
\mathbf{j}_{1}^{T}(0)=-\rho w \beta D_{S} \nabla T .
$$

Comparison of the above with Eq. (39) gives

$$
D_{T}(0)=\beta D_{S}
$$

once again in accord with Eq. (2).

\section{Correction factor}

In retrospect it is unsurprising that the infinite-dilution thermal diffusivity $D_{T}(0)$ given by Eq. (44) is identical to the thermal diffusivity of the pure solvent, Eq. (7). After all, $D_{T}(w)$ possesses the same value for both solute and solvent species and, of course, so too does the limiting value $D_{T}(0)$. Explicitly, were the concentration dependence of $D_{T}(w)$ on the solute concentration $w$ to be known experimentally and was that functional dependence then extrapolated to the infinite-dilution, zero solute concentration limit $w \rightarrow 0$, it would be natural to expect this limiting value, $D_{T}(0)$, to coincide with the thermal diffusivity $D_{T}^{S}$ of the pure, solute-free solvent. Of course this expectation implicitly assumes, inter alia, that the physicochemical inertness criterion is satisfied [52]. In turn, this is tantamount to requiring that the mixture constitutes a thermodynamically ideal solution (whereby one attains Raoult's law in the limit rather than Henry's law [53]).

Because of the various approximations that have entered into the derivation of Eq. (2), we compensate for their possible inaccuracies by inserting an empirical correction factor into that relation, rewriting it as [22]

$$
D_{T}=\lambda \beta D_{S},
$$

in which the dimensionless parameter $\lambda$ is expected to be of $O(1)$ for both liquids and gases, and wherein we have suppressed the affix (0) for notational simplicity.

\section{Agreement with experiment}

Equation (45) for the mixture's binary thermal diffusivity, wherein the solute is either a colloid, a macromolecule, or more generally a molecule much larger in size than that of a solvent molecule, agrees exactly with an empirically proposed equation for $D_{T}$ set forth in Ref. [22]. That accord was tested for a wide variety of liquid and gaseous binary mixtures by establishing whether or not the ratio

$$
\lambda:=\frac{D_{T}^{\exp }}{\beta D_{S}}
$$

of experimental to theoretical thermal diffusivities was indeed of $O(1)$ for these data sets.

Satisfaction of this $O(1)$ requirement, signaling the credibility of our elementary theory [22], was borne out for all of the mixtures examined, involving both liquids and gases. The value of $\lambda$ for the case of liquids was found to be such that $\lambda \geq 1$ in all cases (with but a single exception, where it had the value $\lambda=0.9$, which was regarded as statistically indistinguishable from unity).

In addition to the sporadic and unorganized study of $\lambda$ values in Ref. [22], Hartung, et al. [54] have, inter alia, performed a more systematic study of the viability of Eq. (45) for dilute macromolecular solutions of polystyrene in a variety of different liquid solvents. They found, irrespective of solvent choice, that the value $\lambda=3.7$ produced a very good fit to all of their thermal diffusion data (see their Fig. 3). Their interpretation of this solvent-independent finding was that the nonideality factor $\lambda$ is, at least in this instance, a function solely of the physicochemical nature of the solute. Comparable data for a variety of other polymers would be needed to establish whether this solvent-independent finding might be more widely applicable.

As regards other data in support of our theory we take note of the fact that the thermal diffusivity is predicted to be independent of solute size, e.g., the molecular weight of a polymer in a binary liquid mixture (at least to the extent that $\lambda$ is independent of solute size). In the latter macromolecular-solution case this predicted chain-length independence is well supported by a variety of data covering a range of polymers, molecular weights (except for the very smallest), and chain conformations. This finding is documented by large variety of data in the review paper by Wiegand [[3], Fig. 7] for the case of macromolecules at infinite dilution. At the same time these data, covering different solvents and polymers (polystyrene, polymethylmethacrylate, and polyisobutylene), fall into distinct solvent groupings for the same polymer. This suggests, as in the case of Hartung, et al. [54], that the $\lambda$ value may depend primarily on the solute. This issue, however, needs to be studied more systematically.

\section{E. Reversal in particle migration direction. From hot to cold or cold to hot?}

Yet another manifestation of the theory's success stems from the following considerations. Apart from the general accord displayed by the $\lambda=O(1)$ findings for both liquids and gases, one of the most striking experimental aspects of Eq. (45) stems from the fact that the $\beta$-coefficient for liquids is an algebraically signed parameter, whence the according to our theory the same is true of $D_{T}$. [For (ideal) gases $\beta$ $=1 / T$ is one-signed for all temperatures.] For example, $\beta$ $>0$ for water at temperatures above $4{ }^{\circ} \mathrm{C}$, whereas below that temperature, $\beta<0$. Thus, a colloidal or macromolecular solute particle in a dilute aqueous solution is predicted to migrate from hot to cold at temperatures above $4{ }^{\circ} \mathrm{C}$, with the migration occurring from cold to hot below this temperature. Moreover, according to Eq. (45) this reversal in direction occurring in the neighborhood of $4{ }^{\circ} \mathrm{C}$ holds irrespective of the colloidal particle's physicochemical nature, since the direction-reversal phenomenon is an attribute of the water itself, independent of the solute.

Within experimental error these predictions are borne out by the aqueous experiments of Iacopini, et al. [55], as critically reviewed in Ref. [22], the latter confirming the change 
in migration direction in the neighborhood of $4{ }^{\circ} \mathrm{C}$ for a variety of different solutes [56,57].

With respect to yet another effect of temperature, Eq. (45) also offers a potentially quantitative rationalization of the experimentally observed increase of $D_{T}$ with temperature for both liquids [55,58] and gases [17], as follows from the fact that the thermal expansivities and self-diffusivities of most fluids increase with temperature (see Table V of Ref. [22] for the case of water). Equally worthy of exploration via Eq. (45) are the effects of pressure [59] or of solute molecular weight (in homologous sequences of chemical compounds [60]) on thermal diffusion in liquids and gases [17].

\section{F. Do Marangoni surface-energy gradient stress effects explain the systematic $\boldsymbol{\lambda}>1$ values observed for liquids?}

Virtually all current thermodiffusion theories focus exclusively on liquids (there being no apparent need to address gases owing to the eminently satisfactory Chapman-Enskog theory of thermal diffusion in gases [17]). Those theories concerned with nonionic liquids trace their roots back to the work of both Ruckenstein [18] and Anderson [18], arguing that thermophoretic motion and thermal diffusion in liquids are largely a consequence of surface forces arising from temperature-dependent surface-energy phenomena manifested at the solute-solvent interface, e.g., Marangoni forces [61] stemming from the temperature dependence of the interfacial tension. In the case of ionic solvents, thermodiffusion phenomena are largely regarded as electrokinetic in origin, again focusing on temperature-dependent surface phenomena, e.g., surface charge. None of these contemporary surface-energy gradient-based theories $[7,9,19,20]$ appear to recognize the existence of self-thermophoretic forces of the type advanced here, much less their fundamental role in animating thermodiffusive movement [62].

Moreover, given the relative unimportance of surface forces in gases, these theories are intrinsically incapable of providing a unified theory of thermodiffusion phenomena applicable to both liquids and gases. In addition, even when limited to liquids, lack of knowledge of the some of the uncommon physical parameters appearing in these interfacial theories $[7,9,19,20]$, much less knowledge of their respective temperature dependencies, severely limits the ability of these theories to furnish reliable estimates enabling quantitative comparison with experimental data.

The fact that $\lambda$ was consistently found to be larger than unity for all of the liquid mixtures studied [22,54] suggests that systematic departures from the present solvent-based theory might be a consequence of such surface forces, whose actions over and above the purely molecular selfthermophoretic statistical forces would cause the solute velocity $\mathbf{U}_{1}^{T}(0)$ to be greater than predicted-in turn, furnishing a value of $D_{T}$ exceeding that attributable solely to such forces alone. (This assumes, of course, that the net effect of the surface forces acts in the same direction as the selfthermophoretic forces such as to produce values of $\lambda$ greater, rather than, less than unity.)

The magnitudes of the larger-than-unity $\lambda$ values observed in experiments suggests $[22,54]$ for a given tempera-
TABLE I. Experimental thermal diffusivity of dilute waterpolystyrene (PS) latex mixtures [63].

\begin{tabular}{cc}
\hline \hline $\begin{array}{c}\text { Particle diameter } \\
(\mathrm{nm})\end{array}$ & $\begin{array}{c}\text { Thermal diffusivity } D_{T}(0) \times 10^{12} \\
\left(\mathrm{~m}^{2} \mathrm{~s}^{-1} \mathrm{~K}^{-1}\right)\end{array}$ \\
\hline 105 & 3.63 \\
220 & 3.05 \\
300 & 2.55 \\
398 & 2.01 \\
\hline \hline
\end{tabular}

ture gradient that these surface forces, supposing them to exist, were comparable in strength to those due to selfthermophoresis. However, Marangoni surface forces are size dependent [18], whereas thermophoretic forces are not. As such, a signature of surface forces, were they indeed to be present, would be to render $D_{T}$ [and hence the solute's thermodiffusion velocity $\left.\mathbf{U}_{1}^{T}(0)\right]$ dependent upon solute particle size. This issue, even that of the reliability of the experimental data itself bearing thereon is, however, quite controversial as is evident from the recent studies thereof $[4,12,13]$ discussed below.

Surface-based thermodiffusion theories, such as that of Würger [9], predict an increase in $\left|D_{T}\right|$ with increasing particle size for colloidal particles. However, the thermal diffusion data of Shiundu, et al. [63], as reviewed in Ref. [22] (see Tables II and IV of that paper, as well Table I of the present paper), and covering a wide variety of colloidal particle types and sizes, displays exactly the opposite trend. To further confound the situation, other data [13] show no effect of particle size on thermal diffusivity, whereas still other data [8] support predictions, like that of Würger [9], namely, of a linear increase in thermal diffusivity with particle size. These contentious findings are critically reviewed by Piazza and Parola [[4(b)]]. The uncertain status of the magnitudes (and net directions) of possible macroscopic surface forces relative to molecular self-thermophoretic forces, render it is evident that a rational explanation for the $\lambda \geq 1$ values empirically observed for liquids awaits further developments.

\section{CONCLUSIONS}

\section{A. First-principles derivation of $D_{T}$}

In the case of gaseous mixtures, elaborate theories culminating in explicit and accurate predictions of thermal diffusivities date back to the classic, century-old pioneering analyses by Chapman and Enskog [17]. However, the present self-thermophoretic model appears to be the first to offer a simple and moderately accurate predictive theory for liquid (as well as gaseous) mixtures. We refer here to a firstprinciples formula capable of furnishing, from readily available physical property data, numerical values of $D_{T}$ and, as a bonus, the thermophoretic velocities $\mathbf{U}$ of macroscopic bodies. While there exist a number of other theories for liquids, for example $[9,20]$, none appear to be quantitatively predictive in the absence of a host of difficult-to-come-by physical property data. As such, to the writer's knowledge, none of these theories has been systematically checked against broad 
classes of pertinent experimental data. Even to the extent of merely indicating general trends rather than strict numerical values, these surface energy-based predictions, as discussed, are often inconclusive.

Despite the incompleteness of the present theory's principal result, namely, Eq. (45), with respect to the ability to predict $\lambda$-values a priori, the simplicity of its postulates offers the possibility of eventually incorporating pertinent solute-specific features into the present bare-bones, solventbased model, enabling $\lambda$ to be predicted from first principles.

\section{B. Applicability of the model to both liquids and gases}

Though emphasis thus far has focused on the applicability of Eq. (45) to liquids, it is equally applicable to gases as is evident from the generic nature of its derivation. Current theories explaining and quantifying the phenomenon of thermal diffusion in gases [17] differ profoundly from those for liquids. In contrast, Eq. (45) offers a unified model encompassing both classes of fluids. Typical thermal diffusivity values for liquids are of $O\left(10^{-12}\right)$ and $O\left(10^{-11}\right) \mathrm{m}^{2} \mathrm{~s}^{-1} \mathrm{~K}^{-1}$ for colloidal and macromolecule solutes, respectively, whereas those for gases are of $O\left(10^{-7}\right) \mathrm{m}^{2} \mathrm{~s}^{-1} \mathrm{~K}^{-1}$, with the two thus differing by four or five orders of magnitude. As such, it speaks well of the solidity of its foundations that a single theory, one whose end result is embodied in Eq. (44), or more accurately in Eq. (45), is capable of accommodating both classes of fluids within its jurisdiction. This success is especially gratifying given the extraordinary simplicity of the model relative to the perceived complexity of thermodiffusion phenomena. (Indeed, discussion of these phenomena are often either completely ignored or relegated to obscure locations in standard transport-phenomena and fluid-mechanical textbooks.) The ability to accommodate such disparate $D_{T}$ values within a single model is obviously attributable to the fundamental role played therein by the solvent's selfdiffusivity, a physical attribute not explicitly present in previous theories of thermal diffusion, which focus primarily on the solute rather than the solvent [64].

\section{Macroscopic thermophoretic velocities in single-component fluids}

In addition to the present theory's demonstrated ability to predict binary thermal diffusivities in liquid and gaseous mixtures, the theory also predicts values of the thermophoretic velocities of macroscopic bodies in these fluids. Explicitly the thermophoretic velocity $\mathbf{U}$ of a (nonheatconducting) macroscopic particle of any size or shape in a liquid or gas is predicted to be

$$
\mathbf{U}=-\beta D_{S} \boldsymbol{\nabla} T,
$$

provided that the particle is physicochemically inert relative to its interactions with the fluid in which it is immersed. The question naturally arises as to whether or not the correction factor $\lambda$ should be inserted into this equation, since as shown in Sec. III for the case of gases, where $\beta=1 / T$, the present, uncorrected formula agrees very well with experiment without requiring a $\lambda$ correction factor. Moreover, there are vir- tually no data, reliable or otherwise, pertaining to thermophoretic velocities of particles in liquids owing to the general inability to accurately measure the small speeds encountered.

We have earlier referred to the quantity defined by the expression $\mathbf{U}^{T}(0):=-D_{T}(0) \boldsymbol{\nabla} T$ as being the thermodiffusion velocity of a macroscopic particle, specifically that of the solute particle (molecule) in a dilute binary mixture. However, evidence is equivocal with respect to whether or not the $\mathbf{U}^{T}(0)$ value derived as above from the experimentally measured infinite-dilution thermal diffusivity value $D_{T}(0)$ furnishes the thermophoretic velocity $\mathbf{U}$ of a (macroscopic) particle moving through the pure solvent (much less that of a particle of the same physicochemical constitution as that of the solute employed in the mixture experiment).

Explicitly, if $\lambda$ is defined in terms of thermal diffusivity measurements via Eq. (45), the question is whether or not we should then also write for an isolated macroscopic particle in a fluid-with the particle having nothing whatsoever to do with either mixtures or thermal diffusion-that the particle's thermopheretic velocity is

$$
\stackrel{?}{\mathbf{U}=}-\lambda \beta D_{S} \nabla T .
$$

After all, in the case of gases (for which $\beta=1 / T$ ), we have in Secs. II and III demonstrated insofar as the thermophoresis of an isolated macroscopic particle is concerned that $\lambda=1$. On the other hand, for the only gaseous mixtures whose thermal diffusivities were studied in the context of the present theory (see Table VII of Ref. [22]) the $\lambda$ values derived from thermal diffusion measurements via Eq. (45), namely $D_{T}(0)=\lambda \beta D_{S}$, were found to be $0.25-0.30$, rather than unity.

\section{Thermophoretic velocities in liquids}

An oft-cited, albeit controversial paper by $\mathrm{McNab}$ and Meisen [65] represents the first attempt to directly measure the thermophoretic velocities of individual colloidal particles suspended in liquids. Their experiments were performed on colloidal polystyrene-latex particles dispersed in water and hexane solvents, with both liquids being highly diluted in solute (involving fractional particle volumes of the order of $\left.10^{-5}\right)$. Their experiments involved two sets of spherical particles of highly uniform diameters $d_{p}=1011$ and $790 \mathrm{~nm}$, respectively. Experiments were conducted in the vicinity of room temperature, using temperature gradients between 70 and $300 \mathrm{~K} \mathrm{~cm}^{-1}$.

$\mathrm{McNab}$ and Neisen correlated their measurements via the formula

$$
\mathbf{U}=-\gamma\left(\frac{k}{2 k+k_{p}}\right) v \nabla \ln T,
$$

in which $k$ and $k_{p}$ are the respective fluid and particle thermal conductivities. They found $\gamma=0.26$ to be the "best-fit" value for their data.

Except for the $\gamma$ coefficient, Eq. (49) is identical to Epstein's [43] theoretical formula for the thermophoretic velocity of a heat conducting spherical particle in a gas, where Maxwell's slip coefficient $C_{S}$ (multiplied by a factor of 2) appears in place of $\gamma$ [see Eq. (21) for the nonconducting case]. With regard to its application to liquids, Eq. (49) is 
completely empirical despite its theoretical basis for the case of gases. McNab and Meisen provide literature values of the thermal conductivities and kinematic viscosities of water and hexane as functions of temperature in the vicinity of room temperature, in addition to furnishing the thermal conductivity of the polystyrene particles used in establishing the above correlation.

Was the general constitutive format of their correlation to be even remotely correct, and were their experimentally measured thermophoretic velocities $\mathbf{U}$ deemed reliable, those data could be used go estimate the thermal diffusivity $D_{T}(0)$ of dilute mixtures of polystyrene latex in water at $25^{\circ} \mathrm{C}$ via

the relation $\mathbf{U}=-D_{T}(0) \nabla T$. This calculation furnished the following estimate:

$$
D_{T}(0)=345 \times 10^{-12} \mathrm{~m}^{2} \mathrm{~s}^{-1} \mathrm{~K}^{-1} .
$$

By way of comparison, experimental thermal diffusion data from several independent sources are available for dilute water-polystyrene latex particle mixtures at room temperature, and for several particle sizes. Thus, for $60 \mathrm{~nm}$ diameter particles and a temperature of $25^{\circ} \mathrm{C}$, Iacopini, et al. [55] found that

$$
D_{T}(0)=1.6 \times 10^{-12} \mathrm{~m}^{2} \mathrm{~s}^{-1} \mathrm{~K}^{-1} .
$$

On the other hand, for the same mixture, Shiundu, et al. [63], using thermal field-flow fractionation techniques [42], observed the values noted in Table I for various particle sizes.

By way of comparison (using the values $\beta=0.257$ $\times 10^{-3} \mathrm{~K}^{-1}$ and $D_{S}=2.30 \times 10^{=9} \mathrm{~m}^{2} \mathrm{~s}^{-1}$ for water [22]), Eq. (44) provides the estimate

$$
D_{T}(0)=0.6 \times 10^{-12} \mathrm{~m}^{2} \mathrm{~s}^{-1} \mathrm{~K}^{-1} .
$$

Subject to the $\lambda=O(1)$ accuracy criterion presumably applicable to this correction factor, Eqs. (51) and (52) are consistent with one another, as they are too with the values tabulated in Table I.

However, comparison of these consistent thermal diffusivity values with the McNab-Meisen derived value, Eq. (50), points to a major discrepancy. There are, of course, side issues arising for example from the role of the nonzero thermal conductivity in their formula. Nevertheless, their results would require a $\lambda$ value far in excess of the $O(1)$ values established experimentally in Ref. [22] to hold for a wide variety of similar liquid-phase mixtures. As such it is highly likely that the McNab-Neisen thermophoretic velocity measurements were flawed in some, as yet, unknown manner, e.g., owing to the possibility of natural convection currents. This conclusion regarding serious problems with their data, while tentative, points up yet another controversial element pertaining to current thermodiffusion theories for liquids [2-4].

Using thermal field-flow techniques [42], Regazzetti, et al. [66] measured what they termed the "thermophoretic velocities" of several types of non-Brownian particles in various liquids. However, their particle velocity data were not obtained by tracking the movements of individual particles through the fluid. Rather, their "velocities" were obtained indirectly, without direct observation, even as regards the directions in which their particles moved under the influence of the temperature gradient. Based upon a review of their experimental protocol in Ref. [22], it was concluded that their scheme likely did not objectively measure the true thermophoretic velocities $\mathbf{U}$ of isolated macroscopic particles.

Duhr and Braun [8], pursuing their interest in thermal diffusion measurements using water as the solvent (pertinent to its role in biological processes), performed direct singleparticle tracking measurements involving polystyrene particles for cases where their particles were regarded as being too large $(>500 \mathrm{~nm}$ dia. $)$ to have their thermal diffusivities $D_{T}(0)$ reliably measured by standardized techniques. Their direct, single-particle tracking measurements of the particle's thermophoretic velocity $\mathbf{U}$, when converted into thermal diffusivities via the relation $\mathbf{U}=-D_{T}(0) \boldsymbol{\nabla} T$ yielded values of $D_{T}(0)$ that were consistent with the extrapolation of their presumably reliable thermal diffusion measurements to these larger size particles. In turn, this accord would normally appear to confirm that the thermophoretic velocity of an essentially macroscopic-size colloidal particle in a liquid is indeed given by Eq. (44), at least to within an uncertainty of $O(1)$.

However, Duhr and Braun's experimental protocol, and hence their results and conclusions, have been questioned by Braibanti, et al. [13] as well as by Putnam, et al. [67]. As such, unequivocal experimental confirmation of the proposed thermophoretic velocity formula Eq. (48) for liquids remains elusive, although its validity for gases appears to have been established beyond question in Sec. II and III.

\section{CLOSURE}

\section{A. Terminology}

The term "self-thermophoresis" appears to have first been coined by Golestanian, et al. [68], although the closelyrelated term "self-induced thermophoresis" was used earlier by Williams [69]. However, neither of these prior uses bears on the molecular self-thermophoretic phenomenon described herein. In both of the preceding cases, the prefix "self" refers to an attribute possessed by an individual macroscopic particle - a foreign body introduced into a single-component fluid. In the case of Golestanian, et al. the motion of the foreign body arises from inhomogeneities in its physicochemical surface properties, a particle-specific attribute that comes to the fore as a consequence of the body's hydrodynamic interaction with the fluid's externally imposed temperature field.

Motion in Williams' [69] case is a consequence of heat generation within the interior of an asymmetric particle immersed in an otherwise isothermal fluid, such that the resulting local nonuniform temperature distribution generated by heat transfer from the particle into the surrounding fluid results in the existence of a temperature gradient along the particle's surface. Phoretic motion of the body through the fluid then occurs either as a result of the phenomenon of Maxwell thermal creep [70] or as a result of interfacial tension gradients giving rise to Marangoni forces (or both).

In contrast, our use of the prefix "self" does not refer to the phoretic motion of a macroscopic body possessing an 
inhomogeneous surface attribute. Rather, the term applies to the statistically mean motion of a single molecule, animated by the collective properties of all of the molecules in the system acting thereon. What principally distinguishes the phenomenon of "self-thermophoresis" from that of "thermophoresis" is that the former is a latent or virtual molecular property exclusively of the fluid, a property that in contrast with the above-cited thermophoretic case is not expressed until such time as a foreign body - a macroscopic "tracer" particle-is introduced into the quiescent nonisothermal fluid.

\section{B. Philosophical overview of the modeling of thermodiffusion phenomena in dilute systems. Solute vs solvent as the focus}

For reasons of simplicity most previous attempts at modeling binary thermal diffusion phenomena in liquids has focused on dilute solutions of a solute whose molecules are large enough to be considered as discrete, effectively macroscopic, entities relative to the molecules of the solvent. This enables the latter to be viewed as a hydrodynamic fluid continuum, thereby allowing the forces acting on a discrete, effectively isolated solute particle to be determined by use of joint hydrodynamic and physicochemical arguments. However, despite the fact that the mixture's thermal diffusion coefficient $D_{T}$ has the same value for both solute and solvent, the focus in the modeling process has invariably been on the solute. This approach follows the lead of Einstein [21] who, despite the fact that the mutual diffusion coefficient $D$ appearing in Fick's law is the same for both solute and solvent, also chose to focus on the solute.

The solute's diluteness enables one to focus attention solely on solute-solvent interactions (and implicitly on solvent-solvent intermolecular interactions) while, at the same time, ignoring solute-solute interactions. One next seeks to establish the forces acting on the isolated solute molecule as it wends its way through the otherwise quiescent solvent continuum. In Einstein's isothermal diffusion case this ansatz is manifested by his use of Stokes law. In the thermal diffusion case the ansatz is generally accomplished by an extension of the views of or Ruckenstein [18] or Anderson [18] who, in attempting to explain phoretic phenomena, focus exclusively on surface forces arising from temperature and other field gradients in the solvent bathing the solute molecule. Such has largely been the history of liquid-phase thermal diffusion models to date. Our model is fundamentally different, since it chooses to focus on the solvent rather than the solute. It does so by exploiting the fact that the thermal diffusion coefficient is the same for both solute and solvent.

\section{Concentration effects: Nondilute systems}

The solvent-based approach to modeling binary thermal diffusion phenomena as described in the preceding subsection can, presumably, be extended straightforwardly to the case of nondilute solutions. The physical basis for this extension resides in the fact that even in nondilute systems of colloids or macromolecules the local number density of sol- vent molecules at a point of the mixture continues to greatly exceed that of the higher molecular weight solute molecules owing to their disparate molecular sizes. (Basically, while the weight fraction of solute molecules in the mixture may be large, their mole fraction continues to be very small.) Accordingly, the same molecularly based philosophy as summarized above may be applied equally in both dilute and nondilute systems, wherein the diluteness measure refers to the solute's weight fraction rather than to its mole fraction.

Extension of the preceding analysis to such nondilute systems is nor our goal here. Rather we content ourselves here with simply directing attention to the concentrated macromolecular-solution experiments of Rauch et al. [71]. These authors show, empirically, that a virtually universal correlation exists between a nondilute polymer-solution's thermal diffusivity and the solvent's isothermal tracer selfdiffusivity, albeit in the concentrated mixture. Their work confirms for the nondilute case that the latter, solventspecific coefficient, now a mixture property dependent upon $w$, constitutes the dominant parameter governing the rate of thermal diffusion in macromolecular mixtures.

\section{Solute and size independence of the thermal diffusivity}

The claim that thermal diffusivities $D_{T}$ in dilute, thermodynamically ideal mixtures depend upon only the physical properties of the solvent, independently of the solute's physicochemical nature and molecular size is only approximately true, as is clear from our analysis. Nevertheless, in the case of liquids these conclusions are satisfactorily borne out [to within a disparity of $O(\lambda)$ ] by experimental data for both water and acetonitrile as the solvents, as, respectively, summarized in Tables I, II, and IV of Ref. [22] for the cases of solutes of varied physicochemical natures and diverse sizes. The same degree of accord with observation is also found to hold for gases, as can seen from the experimental data presented in Table VII of the preceding reference, with hydrogen as the solvent and with four different noble gases ranging from helium to xenon as the respective solutes.

\section{E. Difference between the effects of a pressure gradient and a temperature gradient in their roles as driving forces in quiescent nonequilibrium steady-state fluids}

In the course of the present analysis (as well as in a prior paper [24]) it was implicitly supposed that the PlanckEinstein mobility formula (13) holds not only for isothermal fluids, but also in the nonisothermal case. This is not strictly true as shown from the analyses of Landauer [72], van Kampen [73], and many others, e.g., [74,75], all concerned with the form of the Fokker-Planck equation for nonequilibrium steady states, such as occur in the case of steady-state heat conduction, where temperature gradients result in the socalled "blowtorch effect." Because of this phenomenon, in the case of a fluid at rest the equilibrium distribution of matter arising from a pressure gradient in a gravitational field is not strictly equivalent in its effect to the corresponding nonequilibrium distribution in a temperature field. In the context of thermal diffusion phenomena the role of the blowtorch effect has been recognized by Bringuier et al. [10,11] as 
modifying the usual interpretation of the Soret effect as simply representing a balance between thermal diffusion and molecular diffusion. In near-equilibrium systems the temperature dependence of the diffusion coefficient constitutes the main correction to that theory required by the blowtorch effect.

\section{F. Summary and conclusions}

Our analysis contradicts the assertion by Semenov and Schimpf [20] that what is here termed self-thermophoresis is impossible in pure fluids. Negation of their claim is demonstrated here in the form of a simple asymptotic theory showing that self-thermophoresis in single-component fluids is not only possible, but in fact leads to significant new results pertaining to thermal diffusion in gases and liquids-results that are corroborated by experiments.

\section{ACKNOWLEDGMENT}

I am grateful to Professor Jan V. Sengers of the University of Maryland for bringing the Semenov-Schimpf [20] paper to my attention, and for his ongoing encouragement in related matters.

\section{APPENDIX: VOLUME VELOCITY: THE DIFFUSE TRANSPORT OF VOLUME}

As a consequence of the inseparability of volume from mass, mass can neither exist nor be transported through a fluid without being accompanied by a proportional amount of volume. Surprisingly, the converse is not true. Explicitly, volume can be transported diffusively, by purely molecular motion, without the net movement of mass. This occurs in situations where density gradients exist (e.g., as a result of a temperature gradient in a single-component fluid or a species concentration gradient in a mixture whose individual species possess different specific volumes) $[24,29,76]$. It is this phenomenon that gives rise to the existence of a volume velocity $\mathbf{v}_{v} \neq \mathbf{0}$ in a fluid at rest (namely, where its mass velocity $\mathbf{v}_{m}$ $=\mathbf{0})$.

The quantity referred to here as the volume velocity and represented by the symbol $\mathbf{v}_{v}$ is, actually, the flux (density) of volume $\mathbf{n}_{v}$ [25]. However, the dimensions of this flux are volume per unit area per time. Since volume divided by area is length, it is seen that $\mathbf{n}_{v}$ has the dimensions of length per unit time, nominally that of a speed. Consequently, since $\mathbf{n}_{v}$ is a vector, this flux is seen to be equipollent with a vector velocity, namely, $\mathbf{n}_{v}=\mathbf{v}_{v}$. Hence the choice of name and symbol.

In the case of homogeneous fluids at rest, wherein the density $\rho$ is uniform throughout, the volume $V$ occupied by a fluid of mass $M$ is, by definition, related to $M$ though the expression $V=M \hat{v}$, in which $\hat{v}=1 / \rho$ is the specific volume.
By analogy to the latter, it is implicitly, albeit wrongly, assumed in the literature that this same proportionality extends locally to the case of inhomogeneous fluids (for which $\nabla \rho$ $\neq \mathbf{0}$ ). Explicitly, were that to be the case, one would have at

each point of the fluid that $\mathbf{n}_{v}=\mathbf{n}_{m} \hat{v}$, where $\hat{v}$ (or, equivalently, $\rho$ ) refers here to its local value at that point, and where, by definition, the mass flux $\mathbf{n}_{m}$ is related to the fluid's mass velocity $\mathbf{v}_{m}$ by the expression $\mathbf{n}_{m}=\rho \mathbf{v}_{m}$ or, equivalently, by $\mathbf{v}_{m}=\mathbf{n}_{m} \hat{v}$. Thus, were the assumed analogy to hold, it

would follow that $\mathbf{v}_{v}=\mathbf{v}_{m}$, whence the two velocities would merge into one. Expressed alternatively, were one to define a symbol $\mathbf{j}_{v}$ by the expression

$$
\mathbf{n}_{v}=\mathbf{n}_{m} \hat{v}+\mathbf{j}_{v},
$$

it would follow that $\mathbf{j}_{v}=\mathbf{0}$.

By definition, $\mathbf{n}_{m} \hat{v}$ appearing in the above expression constitutes the convective portion of the total volume flux $\mathbf{n}_{v}$, whereas $\mathbf{j}_{v}$ represents its molecular or diffuse portion. It is shown elsewhere that the diffuse volume flux $\mathbf{j}_{v} \neq \mathbf{0}$ in the case of multicomponent fluid mixtures whose speciesspecific volumes differ from one another (such that volume is not conserved). Explicitly, in the case of an isothermal, isobaric, body force-free binary mixture undergoing molecular diffusion owing to species concentration gradients, one has that $\mathbf{j}_{v}=\left(\bar{v}_{1}-\bar{v}_{2}\right) \mathbf{j}_{1}$ (see Sec. 5.1 of Ref. [24]; as well as [29]), where $\bar{v}_{i}$ is the partial specific volume of species $i$, $\mathbf{j}_{1}\left(=-\mathbf{j}_{2}\right)$ is the mass diffusion flux of species 1 , and in which, in the case of thermodynamically ideal solutions, this flux is given by Fick's law as $\mathbf{j}_{1}=-\rho D \nabla w_{1}$ with $w_{i}$ the mass fraction of species $i$.

Viewed as a counterexample to the implicit belief that ?

$\mathbf{n}_{v}=\mathbf{n}_{m} \hat{v}$ under any and all circumstances, whether in singleor multicomponent fluids, isothermal or not, etc., the preceding demonstrates, in general, that $\mathbf{j}_{v} \neq \mathbf{0}$, and hence that $\mathbf{v}_{v}$ $\neq \mathbf{v}_{m}$. Consequently, the fluid's volume velocity is seen to constitute a separate and distinct entity from the fluid's mass velocity. The general existence of a difference in these two velocities can also be rationalized by considering the respective transport equations for mass and volume [25], since as regards the rate of production term appearing in each, mass is a conserved quantity whereas volume, generally, is not.

By definition, a diffuse flux - which arises as a consequence of the collective effects of Brownian motion-is not, itself, accompanied by a (net) mass flux. This shows that volume can be transported through a fluid even when that fluid is at rest. In the case of quiescent single-component fluids, where $\mathbf{v}_{v}=\mathbf{j}_{v} \neq \mathbf{0}$, the volume velocity arises solely as a consequence of the temperature gradient $[38,76,77]$ (i.e., density gradient in an isobaric fluid), as in our thermal diffusion analysis. 
[1] C. Soret, Compt. Rend. 91, 289 (1880).

[2] K. Harstad, Ind. Eng. Chem. Res. 48, 6907 (2009).

[3] S. Wiegand, J. Phys.: Condens. Matter 16, R357 (2004).

[4] R. Piazza, J. Phys.: Condens. Matter 16, S4195 (2004); R. Piazza and A. Parola, ibid. 20, 153102 (2008).

[5] A. Parola and R. Piazza, Eur. Phys. J. E 15, 255 (2004).

[6] A. Parola and R. Piazza, J. Phys.: Condens. Matter 17, S3639 (2005).

[7] S. N. Semenov and M. E. Schimpf, Phys. Rev. E 72, 041202 (2005).

[8] S. Duhr and D. Braun, Phys. Rev. Lett. 96, 168301 (2006).

[9] A. Würger, Phys. Rev. Lett. 98, 138301 (2007); J. Morthomas and A. Würger, J. Phys.: Condens. Matter 21, 035103 (2009).

[10] E. Bringuier and A. Bourdon, J. Non-Equilib. Thermodyn. 32, 221 (2007).

[11] E. Bringuier, Philos. Mag. 87, 873 (2007).

[12] D. Vigolo, G. Brambilla, and R. Piazza, Phys. Rev. E 75, 040401(R) (2007).

[13] M. Braibanti, D. Vigolo, and R. Piazza, Phys. Rev. Lett. 100, 108303 (2008).

[14] P. Reineck, C. J. Wienken, and D. Braun, Electrophoresis 31, 279 (2010).

[15] F. Zheng, Adv. Colloid Interface Sci. 97, 253 (2002).

[16] E. J. Davis, in Encyclopedia of Surface and Colloid Science (Marcel Dekker, New York, 2002), Vol. 4, p. 5365.

[17] S. Chapman and T. G. Cowling, The Mathematical Theory of Non-Uniform Gases, 3rd ed. (Cambridge University Press, Cambridge, England, 1970); J. H. Ferziger and H. G. Kaper, Mathematical Theory of Transport Processes in Gases, NorthHolland, Amsterdam, 1972.

[18] E. Ruckenstein, J. Colloid Interface Sci. 83, 77 (1981); J. L. Anderson, Annu. Rev. Fluid Mech. 21, 61 (1989).

[19] M. E. Schimpf and S. N. Semenov, J. Non-Equilib. Thermodyn. 32, 281 (2007).

[20] S. N. Semenov and M. E. Schimpf, Phys. Usp. 52, 1045 (2009).

[21] A. Einstein, Investigations on the Theory of the Brownian Movement (Dover, New York, 1956).

[22] H. Brenner, Phys. Rev. E 74, 036306 (2006).

[23] L. E. Reichl, A Modern Course in Statistical Physics (University of Texas Press, Austin, 1980); R. Kubo, M. Toda, and N. Hashitsume, Statistical Physics II: Nonequilibrium Statistical Physics, 2nd ed. (Springer, Berlin, 1991).

[24] H. Brenner, Physica A 389, 4026 (2010).

[25] H. Brenner, Physica A 349, 11 (2005).

[26] The issue exists only in the case of single-component fluids. In the case of mixtures, however, it is known [27,28] that these two velocities may differ, albeit in the binary case only where the mixture is both isobaric and isothermal $[28,29]$.

[27] S. R. de Groot and P. Mazur, Non-equilibrium Thermodynamics (North-Holland, Amsterdam, 1962).

[28] H. Brenner, J. Chem. Phys. 132, 054106 (2010)

[29] H. Brenner, J. Chem. Phys. (to be published).

[30] Refinements, such as replicating the same experimental protocol using different size nonBrownian tracer particles, and extrapolating the results to zero size, add to the viability of the measurement. Verifying the measurement using differently shaped particles would also enhance the scheme's credibility and objectivity, as too would be the use of a variety of materials of different physicochemical compositions, etc. The up- stream introduction in both types of experiments allows the disturbance to the fluid created by the laser's intense heat or by the particle's sudden insertion to be dissipated by the time $P$ is reached.

[31] L. D. Landau and E. M. Lifshitz, Fluid Mechanics, 2nd ed. (Butterworth-Heinemann, Oxford, 1987).

[32] G. K. Batchelor, An Introduction to Fluid Dynamics (Cambridge University, Press, Cambridge, England, 1967).

[33] R. B. Bird, W. E. Stewart, and E. N. Lightfoot, Transport Phenomena, 2nd ed. (Wiley, New York, 2002).

[34] H. Brenner and J. R. Bielenberg, Physica A 355, 251 (2005).

[35] M. Holz, S. R. Heil, and A. Sacco, Phys. Chem. Chem. Phys. 2, 4740 (2000).

[36] E. Ruckenstein and H. Liu, Ind. Eng. Chem. Res. 36, 3927 (1997).

[37] H. Brenner, Phys. Rev. E 72, 061201 (2005).

[38] H. Brenner, Physica A 388, 3391 (2009).

[39] H. Brenner, Int. J. Eng. Sci. 47, 902 (2009).

[40] S. Chandrasekhar, Hydrodynamic and Hydromagnetic Stability (Oxford University Press, London, 1961).

[41] R. F. Probstein, Physicochemical Hydrodynamics, 2nd ed. (Wiley, New York, 1994), pp. 14 and 19,.

[42] J. C. Giddings, Unified Separation Science (Wiley, New York, 1991); J. Janca, Philos. Mag. 83, 2045 (2003).

[43] P. S. Epstein, Z. Phys. 54, 537 (1929).

[44] J. C. Maxwell, Philos. Trans. R. Soc. Lond. A 170, 231 (1879).

[45] L. Talbot, R. K. Cheng, R. W. Schefer, and D. R. Willis, J. Fluid Mech. 101, 737 (1980); L. Talbot, Rarefied Gas Dynamics, Part 1, edited by S. S. Fisher (AIAA, New York, 1981), p. 467.

[46] F. B. Pidduck, Proc. London Math. Soc. s2-15, 89 (1917).

[47] See also the subsequent Eq. (37), which shows, similarly, albeit now only for binary fluid mixtures containing small numbers of large-size, inert, colloidal solute particles that the velocity of these effectively macroscopic bodies is exactly the same as the self-thermophoretic velocity of the solvent molecules, despite their vastly different sizes. This theoretical conclusion may be viewed as confirming the empirical relation Eq. (24) since, as originally observed by Einstein in his theory of Brownian motion [21], a solute particle of colloidal dimensions may be viewed, hydrodynamically, as a discrete macroscopic body in the context of, concomitantly, viewing the solvent as a hydrodynamic continuum.

[48] N. Epstein, Powder Technol. 151, 2 (2005); A. Chen, J. R. Grace, N. Epstein, and C. J. Lim, Chem. Eng. Sci. 57, 991 (2002); G. Houghton, Ind. Eng. Chem. Fundam. 5, 153 (1966).

[49] F. Prodi, G. Santachiara, L. DiMatteo, A. Vedernikov, S. A. Beresnev, and V. G. Chernyak, J. Aerosol Sci. 38, 645 (2007); F. Prodi, G. Santachiara, S. Travaini, A. Vedernikov, F. Dubois, C. Minetti, and J. C. Legros, Atmos. Res. 82, 183 (2006); V. G. Chernyak, S. A. Starikov, and S. A. Beresnev, J. Appl. Mech. Tech. Phys. 42, 445 (2001).

[50] Because, as a consequence of the molecule's size, a solute molecule manifests Brownian motion it is implicitly understood that the solute's thermodiffusion velocity $\mathbf{U}_{1}^{T}(0)$, which is viewed as being a macroscopic attribute, is, strictly speaking, the statically mean velocity of the Brownian solute molecules.

[51] This set of equations is necessarily exact at all concentrations 
$w$, irrespective of thermodynamic ideality, since they constitute the respective definitions of $D_{T}$ and $D$.

[52] A related issue concerns the fact that a real macroscopic particle possesses a nonzero thermal conductivity, say $k_{p}$, whereas in order to assure "inertness" of the solute we have implicitly required that $k_{p}=0$, thus eliminating the possibility of thermal exchange between particle and fluid. As a nonzero value of the ratio $k_{p} / k_{S}$ is known to affect the thermophoretic velocity of a particle in a gas [34,43], a similar effect would likely arise in the case of liquids too.

[53] R. M. Rosenberg and W. L. Peticolas, J. Chem. Educ. 81, 1647 (2004).

[54] M. Hartung, J. Rauch, and W. Köhler, J. Chem. Phys. 125, 214904 (2006).

[55] S. Iacopini, R. Rusconi, and R. Piazza, Eur. Phys. J. E 19, 59 (2006).

[56] A striking pair of side-by-side photographs graphically illustrating changes in the direction of movement of DNA molecules in water at the respective temperatures of $3{ }^{\circ} \mathrm{C}$ and $20{ }^{\circ} \mathrm{C}$ appears in the paper by Duhr and Braun [[57], see their Fig. 1]. These pictures show, literally, that a transition in the DNA migration direction must have occurred somewhere between these two temperatures.

[57] S. Duhr and D. Braun, Proc. Natl. Acad. Sci. U.S.A. 103, 19678 (2006).

[58] A. Würger, Langmuir 25, 6696 (2009).

[59] D. R. Caldwell, Deep-Sea Res. 21, 369 (1974); J. Phys. Chem. 77, 2004 (1973).

[60] A. Leahy-Dios and A. Firoozabadi, J. Phys. Chem. B 111, 191 (2007).

[61] D. A. Edwards, H. Brenner, and D. T. Wasan, Interfacial Transport Processes and Rheology (Butterworth-Heinemann, Boston, 1991).

[62] However, some researchers [9] regard pressure gradient-type forces stemming from gradients in the so-called osmoticpressure $\Pi=n k_{b} T$ as contributing to thermal diffusion, while, however, dismissing such forces as being negligible compared with surface forces.

[63] P. M. Shiundu, P. S. Williams, and J. C. Giddings, J. Colloid Interface Sci. 266, 366 (2003).

[64] However, as discussed in Ref. [54] several of the solute-based theories successfully correlate $D_{T}$ inversely with the solvent viscosity $\eta$. As such, were one to accept the applicability of the Stokes-Einstein equation to the solvent molecules, thus rendering $D_{S}$ inversely proportional to $\eta$, modulo the proportional factor, these viscosity-based correlations would possess features similar to ours, albeit only for gases or liquids, separately, but not for both together.

[65] G. S. McNab and A. Meisen, J. Colloid Interface Sci. 44, 339 (1973).

[66] A. Regazzetti, M. Hoyos, and M. Martin, J. Phys. Chem. B 108, 15285 (2004); Anal. Chem. 76, 5787 (2004).

[67] S. A. Putnam, D. G. Cahill, and G. C. L. Wong, Langmuir 23, 9221 (2007).

[68] R. Golestanian, T. B. Liverpool, and A. Adjari, New J. Phys. 9, 126 (2007).

[69] M. M. R. Williams, IMA J. Appl. Math. 36, 275 (1986).

[70] H. Brenner, Phys. Fluids 21, 053602 (2009).

[71] J. Rauch, M. Hartung, A. F. Privalov, and W. Köhler, J. Chem. Phys. 126, 214901 (2007).

[72] R. Landauer, Phys. Rev. A 12, 636 (1975).

[73] N. G. van Kampen, IBM J. Res. Dev. 32, 107 (1988); Superlattices Microstruct. 23, 559 (1998).

[74] M. E. Widder and U. M. Titulaer, Physica A 154, 452 (1989).

[75] A. M. Jayannavar and M. C. Mahato, Pramana, J. Phys. 45, 369 (1995).

[76] H. Brenner, Physica A 389, 1297 (2010).

[77] H. Brenner, Int. J. Eng. Sci. 47, 930 (2009). 